\title{
Sum rules for total hadronic widths of mesons
}

\author{
Michał Majewski \\ Dept. of Theoretical Physics, Univ. of Lodz \\ Pomorska 149/153, 90-236 Łódź, Poland
}

\begin{abstract}
Mass sum rules for meson multiplets derived from exotic commutators may be written for complex masses. Then the real parts give the well known mass formulae (GM-O, Schwinger, Ideal) and the imaginary ones give the corresponding sum rules for total hadronic widths. The masses and widths of the meson nonets submit to a definite orders. It thus follows that tables of the meson nonets should include information about masses, widths and the orders as well as the mixing angle. The width sum rule for the nonet complying with Schwinger mass formula may be depicted as a straight line in the $(m, \Gamma)$ plane. It is easily verifiable and satisfied better for high mass nonets.
\end{abstract}

\section{Introduction}

The particle width is one of its main characteristics as much important as mass and discreet quantum numbers. It tells us something different than the mass and sometimes it may tell more: the widths of the particles with similar masses may differ by many orders; then the widths inform us first which interactionstrong, electromagnetic or weak is responsible for their decay. For hadronic decays the differences are not so big, but usually are of the same order as mass differences. Therefore they merit attention.

The difficulty with the widths within the meson multiplet is that they are in a sense accidental. Indeed, selection rules may suppress more or less the decay of a particular particle thus destroying any given regularity. Such an effect should be especially transparent in low mass multiplets where for some particle two-body decays are forbidden and many-body decays are suppressed (e.g. $\omega$ meson). For more massive multiplets, where many decay channels are opened, we may expect better agreement. However the prediction may be interesting in any case.

\section{Sum rules for nonets}

The approach is based on the technique of exotic commutators [1]. The following system of mass sum rules for a nonet has been obtained [2]:

$$
\begin{aligned}
l_{1}^{2}+l_{2}^{2} & =1 \\
l_{1}^{2} z_{1}+l_{2}^{2} z_{2} & =\frac{1}{3} a+\frac{2}{3} b\left(\equiv z_{8}\right)
\end{aligned}
$$




$$
\begin{aligned}
l_{1}^{2} z_{1}^{2}+l_{2}^{2} z_{2}^{2} & =\frac{1}{3} a^{2}+\frac{2}{3} b^{2} \\
l_{1}^{2} z_{1}^{3}+l_{2}^{2} z_{2}^{3} & =\frac{1}{3} a^{3}+\frac{2}{3} b^{3}
\end{aligned}
$$

Here $a, K, z_{1}, z_{2}$ stand for the mass squared of the isotriplet, isodublet and isoscalar physical mesons respectively $\left(z_{1}<z_{2}\right.$ by choice), $z_{8}$ is the GM-O mass squared, $b=2 K-a$ and the real coefficients $l_{1}, l_{2}$ are introduced by the equation: $\left|z_{8}\right\rangle=l_{1}\left|z_{1}\right\rangle+l_{2}\left|z_{2}\right\rangle$. The known mass formulae for a nonet follow from eqs. (1) $-($ (1).

Eqs. (11)-(14) may be considered for complex masses $\hat{M}^{2}=\hat{m}^{2}-i \hat{m} \hat{\Gamma} . \hat{M}^{2}$ is now nonhermitean, but it can be diagonalized and has orthogonal eigenfunctions. We use for the masses squared the notations: $a, K, x_{1}, x_{2}, x_{8}$, $b$ and for the appropriate imaginary parts the notations: $\alpha, \kappa, y_{1}, y_{2}, y_{8}$, $\beta\left(=2 \kappa-\alpha=\sqrt{(b)} \Gamma_{b}\right)$. The coefficients $l_{1}, l_{2}$ are complex numbers, but $l_{1}^{2}, l_{2}^{2}$, which are their modula squared, are real.

The real parts of the masses squared satisfy usual mass formula and the imaginary ones give the sum rules for the widths.

For GM-O nonet (follows from eqs. (1)), (2)) we find

$$
l_{1}^{2}=\frac{x_{2}-x_{8}}{x_{2}-x_{1}}, \quad l_{2}^{2}=\frac{x_{8}-x_{1}}{x_{2}-x_{1}}, \quad \frac{y_{2}-y_{8}}{x_{2}-x_{8}}=\frac{y_{8}-y_{1}}{x_{8}-x_{1}} .
$$

For Schwinger nonet (follows from eqs. (1)-(3)) we have

$$
\frac{y_{2}-y_{8}}{x_{2}-x_{8}}=\frac{y_{8}-y_{1}}{x_{8}-x_{1}}=\frac{\beta-\alpha}{b-a}
$$

and separate equations for the real and imaginary parts of the mass operator:

$$
\begin{aligned}
\left(a-x_{1}\right)\left(a-x_{2}\right)+2\left(b-x_{1}\right)\left(b-x_{2}\right) & =0 \\
\left(\alpha-y_{1}\right)\left(\alpha-y_{2}\right)+2\left(\beta-y_{1}\right)\left(\beta-y_{2}\right) & =0 .
\end{aligned}
$$

For these nonets only two mass orders are allowed and two widths orders for each of them:

$$
\begin{array}{cccc}
a<x_{1}<b<x_{2} ; & \alpha>y_{1}>\beta>y_{2} ; & \text { or } & \alpha<y_{1}<\beta<y_{2} \\
x_{1}<a<x_{2}<b ; & y_{1}>\alpha>y_{2}>\beta ; & \text { or } & y_{1}<\alpha<y_{2}<\beta .
\end{array}
$$

Both mass orders are observed [3].

Some of the well established Schwinger nonets are collected in the Table 1.

For the Ideal nonet (folows from eqs. (11) - (4)) we have

$$
x_{1}=a, \quad x_{2}=b, \quad y_{1}=\alpha, \quad y_{2}=\beta .
$$

If we apply eqs. (1)-(3) to the octet states $\left(l_{1}^{2}=1, l_{2}^{2}=0\right)$ we get degenerate octet (all mases and widths identical). Degenerate multiplets (octets(?), nonets(?)) do exist. They are shown in the Table 2 .

The formula (6) shows that the points $\left(m^{2}, m \Gamma\right)$ of the Schwinger nonet states lie on a straight line in the $\left(m^{2}, m \Gamma\right)$ plane and consequently the poins $(m, \Gamma)$ lie on the straight line in the plane $(m, \Gamma)$. The slope of this line is indefinite. Mass-width diagram for the nonet $2^{++}$is shown on the Figure 1. For the nonet $1^{--}$the agreement is worse, for the nonet $3^{--}$it is quite good. 
Table 1: Some well established nonets of mesons (masses and widths in MeV)

\begin{tabular}{|c|c|c|c|c|c|}
\hline$J^{P C}$ & $\begin{array}{l}m_{K} \\
\Gamma_{K}\end{array}$ & $\begin{array}{l}m_{a} \\
\Gamma_{a}\end{array}$ & $\begin{array}{l}m_{1} \\
\Gamma_{1}\end{array}$ & $\begin{array}{c}m_{b} \\
(2 \kappa-\alpha) m^{-1}\end{array}$ & $\begin{array}{l}m_{2} \\
\Gamma_{2}\end{array}$ \\
\hline multiplet & $\theta^{G M O}$ & \multicolumn{2}{|c|}{ mass order } & \multicolumn{2}{|c|}{ width order } \\
\hline $1^{--}$ & \multirow{3}{*}{$893.88 \pm 0.26$} & \multirow{3}{*}{$\begin{array}{l}769.3 \pm 0.8 \\
150.2 \pm 0.8\end{array}$} & \multirow{3}{*}{$\begin{array}{c}782.57 \pm 0.12 \\
8.44 \pm 0.09\end{array}$} & \multirow{3}{*}{$\begin{array}{c}1.0031 \pm 0.0011 \\
-24.8 \pm 2.1\end{array}$} & \multirow{3}{*}{$\begin{array}{c}1019.417 \pm 0.014 \\
4.458 \pm 0.032\end{array}$} \\
\hline$\bullet \rho(770)$ & & & & & \\
\hline $\begin{array}{l}\bullet K^{*}(892) \\
\bullet \omega(782)\end{array}$ & & & & & \\
\hline • $\Phi(1020)$ & \multicolumn{3}{|l|}{$(39.28 \pm 0.16)^{\circ}$} & \multicolumn{2}{|c|}{$\alpha>y_{1}>\beta>y_{2}$} \\
\hline $2^{++}$ & \multirow{2}{*}{ 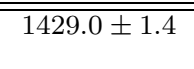 } & \multirow{2}{*}{$\overline{1318.0 \pm 0.6}$} & \multirow[t]{2}{*}{ 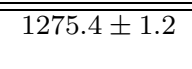 } & \multirow{2}{*}{$\overline{1532.0 \pm 3.1}$} & \multirow[t]{2}{*}{$151525 \pm 5$} \\
\hline$\bullet a_{2}(1320)$ & & & & & \\
\hline $\begin{array}{l}\text { - } K_{2}^{*}(1430) \\
\text { - } f_{2}(1270)\end{array}$ & $103.8 \pm 4.0$ & $107 \pm 5$ & \multirow[t]{2}{*}{$185.1_{-2.6}^{+3.4}$} & $101.5 \pm 11.9$ & $76 \pm 10$ \\
\hline - $f_{2}^{\prime}(1525)$ & $\left(30.67_{-1.72}^{+1.56}\right)^{\circ}$ & $x_{1}<a<x_{2}<b$ & & \multicolumn{2}{|c|}{$y_{1}>\alpha>y_{2}>\beta$} \\
\hline $3^{--}$ & $1776 \pm 7$ & $1691 \pm 5$ & $1667 \pm 4$ & $1857 \pm 11$ & $1854 \pm 7$ \\
\hline $\begin{array}{l}\text { - } \rho_{3}(1690) \\
\bullet-K_{3}^{*}(1780) \\
\text { - } \omega_{3}(1670)\end{array}$ & $159 \pm 21$ & $161 \pm 10$ & $168 \pm 10$ & $158 \pm 53$ & $87_{-23}^{+28}$ \\
\hline$\bullet \Phi_{3}(1850)$ & \multicolumn{3}{|c|}{$x_{1}<a<x_{2}<b$} & \multicolumn{2}{|c|}{$y_{1}>\alpha>y_{2}>\beta$} \\
\hline $1^{++}$ & 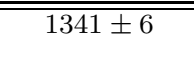 & $\overline{1230 \pm 40}$ & $1281.9 \pm 0.6$ & $1420 \pm 0.012$ & $\overline{1426.3 \pm 1.1}$ \\
\hline $\begin{array}{l}\cdot a_{1}(1260) \\
-K_{A} \\
\cdot f_{1}(1285)\end{array}$ & $134 \pm 16$ & $250 \div 600$ & $24.0 \pm 1.2$ & $-447 \div 89$ & $55.5 \pm 2.9$ \\
\hline - $f_{1}(1420)$ & $35.26^{\circ} \div 41.00^{\circ}$ & $a<x_{1}$ & $<b<x_{2}$ & $\alpha>y_{1}$ & $\beta>y_{2}$ \\
\hline $1^{+-}$ & $1322 \pm 6$ & $1229.5 \pm 3.2$ & $1170 \pm 20$ & $1414 \pm 9$ & $1386 \pm 19$ \\
\hline $\begin{array}{l}\cdot b_{1}(1235) \\
\bullet K_{B} \\
\bullet h_{1}(1170)\end{array}$ & $135 \pm 17$ & $142 \pm 9$ & $360 \pm 40$ & $130 \pm 40$ & $91 \pm 30$ \\
\hline$\bullet h_{1}(1380)$ & $0 \div 35.26^{\circ}$ & $x_{1}<0$ & $<x_{2}<b$ & $y_{1}>0$ & $y_{2}>\beta$ \\
\hline
\end{tabular}

Table 2: Degenerate multiplets (masses and widths in $\mathrm{MeV}$ )

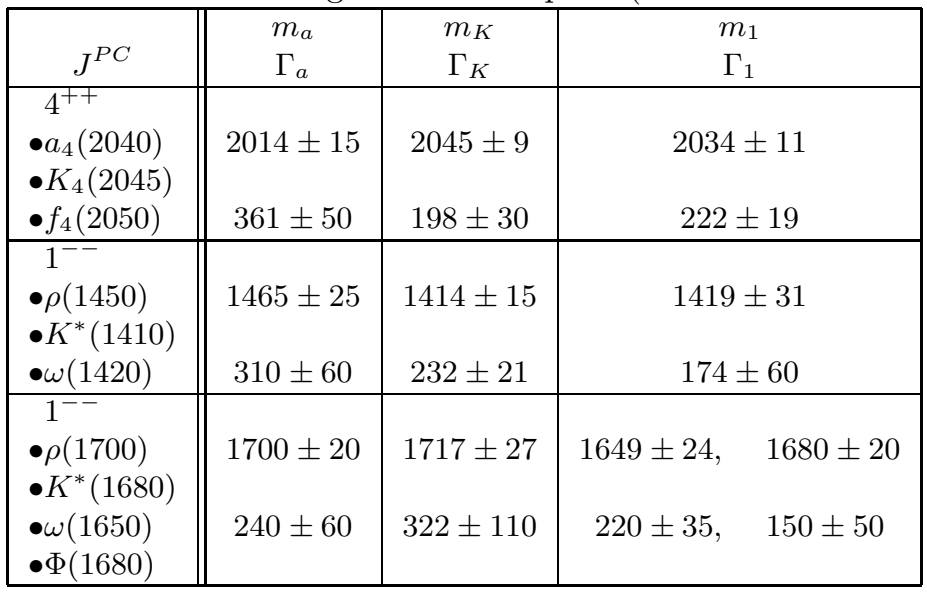




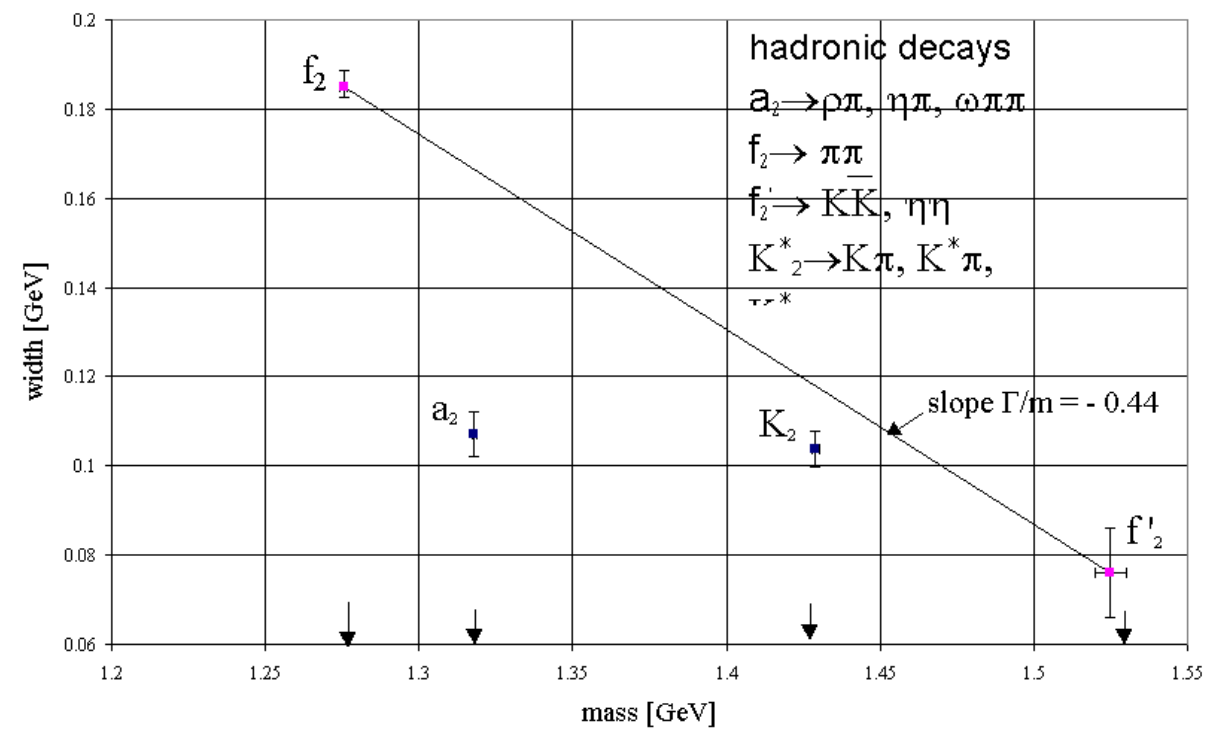

Figure 1: Mass-width diagram of $2^{++}$mesons-mass order: $x_{1}<a<x_{2}<b$

\section{Acknowledgments}

Valuable discusions with Profs. S.B. Gerasimov, P. Kosiński and V.A. Meshcheryakov are kindly acknowledged.

\section{References}

[1] S.Oneda, K.Terasaki Progr. Theor. Phys. Suppl. 82 (1985)

[2] M.Majewski and W.Tybor Acta Physica Polonica B15 (1984) 267

[3] Particle data Group Eur. Phys. J.C15 (2000) 1 